\title{
Exact Solution of the relativistic Schroedinger equation for the central potential $V(r)=\left(-\frac{a}{r}+\frac{b}{\sqrt{r}}\right)$
}

\author{
Dr. V.K. Srivastava, (Late) Prof. S.K. Bose \\ Department of Physics D.D.U. Gorakhpur University, Gorakhpur - 273009, U.P., India
}

\begin{abstract}
A set of exact solutions of the relativistic Schroedinger equation for central potential $(r)=\left(-\frac{a}{r}+\frac{b}{\sqrt{r}}\right.$ ), where $a$ and $b$ are parameters of the given potential are to be obtained by using a suitable ansatz. For each solution, a separate relation interrelating the parameters of the potential and the orbital angular momentum quantum no. $l$. The eigenfunctions obtained here are normalizable. The fractional power potential is relevant in connection with quark model of hadrons and some other branches of physics like particle and nuclear physics.
\end{abstract}

Keywords: Schroedinger equation, fractional Central potential, Exact solution.

\section{Introduction}

One of the important issues of quantum mechanics is to solve the relativistic Schroedinger equation for the potentials of physical interest. Unfortunately, however, only for a few potentials, the Schroedinger equation is found to be exactly solvable. But in recent years, a lot of attention has been paid towards obtaining exact solution of the Schroedinger equation for the potentials of physical importance [1-7,10,11]. Today, it is although possible to solve the relativistic Schroedinger equation for given potential to a desired degree of accuracy with the help of modern computers. An exact solutions, however, have an aesthetic appeal. Further, the exact solutions can serve as benchmark to test the accuracy of various nonperturbative methods.

In the present paper, we obtain a set, consisting no. of solutions which consists eigenvalues and corresponding eigenfunctions of the schroedinger equation for the central potential $V(r)=\left(-\frac{a}{r}+\frac{b}{\sqrt{r}}\right)$, by using suitable ansatz in the form of a series in $r$. This solutions hold good when for each solution, a separate relations, interrelating the parameters of the potential and the orbital angular momentum quantum no. $l$ is satisfied. In such exact solutions, eigenfunction(s) are so normalizable. Such central potential is much more useful in study of interquark potential of the hadron physics and may be useful in others areas of physics $[8,9]$.

\section{Schroedinger equation and exact solution}

For exact solution of the relativistic Schroedinger equation, the central potential is

$$
V(r)=\left(-\frac{a}{r}+\frac{b}{\sqrt{r}}\right)
$$

Where $\mathrm{a}$ and $\mathrm{b}$ are parameters of potential.

Consider a relativistic Schroedinger equation (for convenience, take $\hbar=1=c$ ) and is given by

$$
\left(-\nabla^{2}+m^{2}\right) \Psi=[E-V(r)]^{2} \Psi
$$

Here $\nabla^{2}=$ Laplacian operator in spherical polar co-ordinates $(r, \theta, \phi)$

$m=$ mass of particle

$$
\nabla^{2}=\left[\frac{1}{r^{2}} \frac{\partial}{\partial^{2}}\left(r^{2} \frac{\partial}{\partial r}\right)+\frac{1}{r^{2} \operatorname{Sin} \theta} \frac{\partial}{r \theta}\left(\operatorname{Sin} \theta \frac{\partial}{\partial \theta}\right)+\frac{1}{r^{2} \operatorname{Sin} \theta} \frac{\partial^{2}}{\partial \Phi^{2}}\right]
$$

$\hbar=\frac{h}{2 \pi}$

$h=$ Planck's constant

$E=$ total energy of particle

and $\Psi=$ Normalised wave function

The radial part of the Schroedinger equation for the given potential is

$$
\left[\left\{E-\left(-\frac{a}{r}+\frac{b}{\sqrt{r}}\right)\right\}^{2}-m_{o}^{2}+\frac{1}{r^{2}} \frac{d}{d r}\left(r^{2} \frac{d}{d r}\right)-\frac{l(l+1)}{r^{2}}\right] R(r)=0
$$

Using an ansatz, to solve second order differential equation (3), take

$$
R(r)=\exp \{\alpha r+\beta \sqrt{r}\} \sum_{n=0}^{\infty} a_{n} r^{\frac{n}{2}+v}
$$

where $\alpha, \beta$ and $v$ are constants to be chosen suitably later. 
Exact Solution of the relativistic Schroedinger equation for the central potential $V(r)=\left(-\frac{a}{r}+\frac{b}{\sqrt{r}}\right)$

Using equation (4) in equation (3)and on equating coefficient of $r^{\frac{n}{2}-1+v}$ on both sides, then we have

$$
\begin{aligned}
& A_{n}^{v} a_{n}+B_{n+1}^{v} a_{n+1}+C_{n+2}^{v} a_{n+2}=0 \\
& \text { where } A_{n}^{v}=\beta^{2}+2 E a+(n+2 v+2) \alpha+b^{2} \\
& \beta_{n}^{v}=-2 a b+\left(n+2 v+\frac{3}{2}\right) \beta \\
& \text { and } C_{n}^{v}=\left(\frac{n}{2}+v\right)\left(\frac{n}{2}+v+1\right)-l(l+1)+a^{2}
\end{aligned}
$$

and we have take $\alpha^{2}=m_{0}^{2}-E^{2}$ and $\alpha \beta=E b$, then we should choose

$$
\alpha=-\sqrt{m_{0}^{2}-E^{2}}
$$

So that $R(r)$ is finite for $r \rightarrow \infty$

Now, if $a_{0}$ is the first nonvanishing coefficient in the equation (4), we should have

$$
C_{0}^{v}=0 \Rightarrow v=v_{ \pm}=\frac{1}{2} \pm\left\{\left(l+\frac{1}{2}\right)^{2}-a^{2}\right\}^{\frac{1}{2}},\left(\left\{\left(l+\frac{1}{2}\right)^{2}-a^{2}\right\} \geq 0\right) \ldots \ldots
$$

In which only $v=v_{+}$is physically acceptable.

Again if $a_{p}$ is the last non vanishing coefficient in the equation (4),

We should have $a_{p+1}, a_{p+2}=0$, then the reccurrence relation (5) gives

$$
\begin{aligned}
A_{p}^{v}=0 \Rightarrow\left[\left(b^{2}+2 a E_{p}^{l}\right)\left\{m_{0}^{2}-\left(E_{p}^{l}\right)^{2}\right\}+b^{2}\left(E_{p}^{l}\right)^{2}\right] \\
=\left(p+v_{+}+2\right)^{2} \cdot\left\{m_{0}^{2}-\left(E_{p}^{l}\right)^{2}\right\}^{3}
\end{aligned}
$$

Also for a nontrivial solution, the coefficients $A_{n}^{v}, B_{n}^{v}$ and $C_{n}^{v}$ in the equation (4) must satisfy the determinant relation

$$
\left|\begin{array}{cccccc}
B_{0}^{v} & C_{1}^{v} & \ldots & \ldots & \\
& A_{0}^{v} & B_{1}^{v} & C_{2}^{v} & & 0 \\
\ldots & \ldots & \ldots & \ldots & & \ldots \\
\ldots & \ldots & \ldots & \ldots & & \ldots \\
\ldots & \ldots & \ldots & \ldots & & \ldots . \\
0 & \ldots & \ldots & & A_{p-1}^{v} & B_{p}^{v}
\end{array}\right|=0
$$

Now from equation(10), different exact solution can be generated by putting $p=0,1,2 \ldots \ldots$ etc.

Thus, we have two cases:

Case-I: For $\mathrm{p}=0$, In this case equation (9) gives

$$
\left[\left(E_{0}^{l}\right) b^{2}+\left(2 E_{0}^{l} a+b^{2}\right) m_{0}^{2}-\left(E_{0}^{l}\right)^{2}\right\}^{2}=4\left(v_{+}+1\right)^{2}\left\{m_{0}^{2}-\left(E_{0}^{l}\right)^{2}\right\}^{3}
$$

Equation (11) gives energy eigenvalue and is given by

$$
E_{0}^{l}=m_{0}\left\{1+\frac{a^{2}}{\left(v_{+}+1\right)^{2}}\right\}^{-\frac{1}{2}}
$$

Using equation (11) and (12), we find the interrelation between the parameters $\mathrm{a}$ and $\mathrm{b}$ and is given by

$$
\begin{aligned}
\left\{b^{2}+\frac{a^{2} b^{2}}{\left(v_{+}+1\right)^{2}}\right. & \left.+\frac{2 a^{3} m_{0}}{\left(v_{+}+1\right) \sqrt{a^{2}+\left(v_{+}+1\right)^{2}}}\right\}^{2} \\
& =\frac{4}{\left(v_{+}+1\right)^{2}}\left\{\frac{m_{0}^{2} a^{6}}{\left(v_{+}+1\right)^{2}+m^{2}}\right\}
\end{aligned}
$$

Equation (13) gives the interrelation between the parameters a and $\mathrm{b}$ of central potential and orbital angular momentum quantum no. $l$.

The complete eigenfunction in this case is given by

$$
\Psi_{0, l, \mathrm{~m}}(\mathrm{r}, \theta, \Phi)=\exp \left\{-\frac{\mathrm{am}_{\mathrm{o}} \mathrm{r}}{\sqrt{\mathrm{a}^{2}+\left(v_{+}+1\right)^{2}}}-\frac{2 \mathrm{~b}}{\mathrm{a}}\left(v_{+}+1\right) \mathrm{r}^{\frac{1}{2}}\right\} a_{o} r^{v_{+}} Y_{l}^{m}(\theta, \Phi) \text {. }
$$

Here $Y_{l}^{m}(\theta, \Phi)=$ spherical harmonics

Equation(14) provides the quantum nature of hadrons.

Case II: For $\mathrm{p}=1$, In this case, equation (9) gives

$$
\left[\left(E_{1}^{l}\right)^{2} b^{2}+\left(2 E_{1}^{l} a+b^{2}\right)\left\{m_{0}^{2}-\left(E_{1}^{l}\right)^{2}\right\}\right]=\left(2 v_{+}+3\right)^{2}\left\{m_{0}^{2}-\left(E_{1}^{l}\right)^{2}\right\}^{3} .
$$

The condition, on the other hand, gives 


$$
\begin{gathered}
\left.b^{2}\left\{\left(v_{+}+\frac{3}{4}\right) \frac{E_{1}^{l}}{\sqrt{m_{0}^{2}-\left(E_{1}^{l}\right)^{2}}}+a\right\}\left\{v_{+}+\frac{5}{4}\right) \frac{E_{1}^{l}}{\sqrt{m_{0}^{2}-\left(E_{1}^{l}\right)^{2}}}+a\right\} \\
=\left(v_{+}+\frac{3}{4}\right) \sqrt{m_{0}^{2}-\left(E_{1}^{l}\right)^{2}}
\end{gathered}
$$

(On using equations $6 \mathrm{a}, 6 \mathrm{~b}, 6 \mathrm{c}$ and 7 )

Now, equation (15) and (16) can be used to find the energy eigenvalue and an interrelation between the parameters $\mathrm{a}$ and $\mathrm{b}$ of the central potential and the orbital angular momentum quantum no. $l$. The complete eigenfunctions, for $\mathrm{p}=1$ is given by

$$
\Psi_{1, l, \mathrm{~m}}(\mathrm{r}, \theta, \Phi)=\exp \left[\left\{-\sqrt{\mathrm{m}_{\mathrm{o}}^{2}-\left(E_{1}^{l}\right)^{2}}\right\} \mathrm{r}-\left\{\frac{2 E_{1}^{l} b}{\sqrt{\mathrm{m}_{\mathrm{o}}^{2}-\left(E_{1}^{l}\right)^{2}}}\right\} r^{\frac{1}{2}}\right] r^{v_{+}}\left(a_{o}+a_{1} r^{\frac{1}{2}}\right) Y_{l}^{m}(\theta, \Phi)
$$

The constants $a_{0}$ and $a_{1}$ are related as

$$
\left\{m^{2}-\left(E_{1}^{l}\right)^{2}\right\} a_{0}-2\left\{a b \sqrt{m_{0}^{2}-\left(E_{1}^{l}\right)^{2}}+\left(v_{+}+\frac{5}{4}\right)\left(E_{1}^{l}\right) b\right\} a_{1}=0
$$

Here $m_{o}=$ rest mass of particle.

Continuing this way, we can generate a set of exact solutions for higher values of $\mathrm{p}$.

\section{Results and Discussion}

The explicit expressions of energy eigenvalue and eigenfunction are obtained for each solution. These solutions are valid when for, in general, each solutions has an interrelation between the parameters of the potential and the orbital angular momentum quantum number $l$ is satisfied. These solution, besides having an aesthetic appeal, can be used as benchmark to test the accuracy and reliability of non perturbative methods, which some times yield wrong result, of solving the Schroedinger equations. The central potential considered in this problem may be relevant in study of quark model of hadrons $[8,9]$.

The central irregular singular potentials $V(r)$ for which $\lim _{r \rightarrow 0} r^{2} V(r) \rightarrow \infty$ are mathematically more difficult to treat. A set consisting of eigenvalues and the corresponding eigenfunctions of exact solution of the Schroedinger equation is obtained for the fractional central potential.

\section{Conclusions}

The exact bound state solutions of relativistic Schroedinger equation for central potential $V(r)=$ $\left(-\frac{a}{r}+\frac{b}{\sqrt{r}}\right)$ by using a suitable ansatz have been obtained. The exact solutions consists eigenvalues and corresponding eigenfunctions with an interrelation between parameters $\mathrm{a}$ and $\mathrm{b}$ of potential and the orbital angular momentum quantum number $l$. The eigenfunction which are obtained in closed form, are also square integrable. Such solution of relativistic Schroedinger equation plays most important role in nuclear and particle physics and especially in quarkonium physics (study of quark model of hadrons).

\section{Acknowledgement}

The author gratefully acknowledges Prof. D.C. Srivastava, Ex. HOD, Department of Physics, DDU Gorakhpur University,Gorakhpur for encouragement and Prof. H.C. Prasad for giving necessary suggestions.

\section{References}

[1]. Singh V, Biswas S N and Datta K, Phys Rev., 18D (1978) 1901

[2]. Flessas G P, Phys. Lett., 83A (1981) 121

[3]. Varma V S, J. Phys., 14A (1981) L 489

[4]. Bose S K, J. Math. and Phys. Sc., 26 (1992) 129

[5]. Chhajlany S C and Malnev V N, J. Phys. , 23A (1990) 3711

[6]. Singh L A, Singh S P and Singh KD, Phys. Lett. , 148A (1990) 389

[7]. Dutra A D S and Filho HB, Phys. Rev. , 44A (1991) 4721

[8]. Song X, J. Phys. , 17 (1979) 49

[9]. Stillinger F H, J.Math. Phys., 20 (1979) 1891

[10]. Srivastava V K and Bose S K, Indian J. Pure \& App. Phys., 47 (2009) 547

[11]. Srivastava V K and Bose S K, GSRJ, 3 (2017) 16 